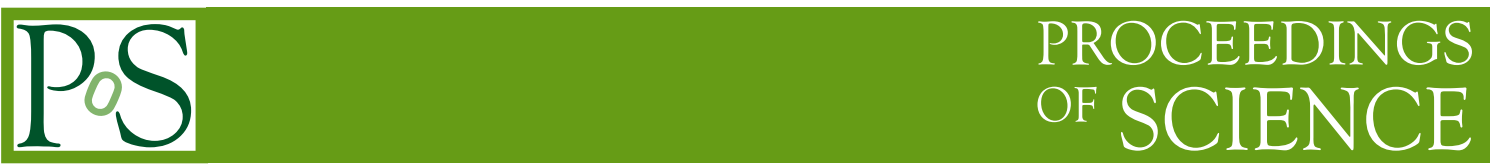

\title{
DAMA/LIBRA results
}

\section{Rita BERNABEI, ${ }^{a}$ Pierluigi BELLI,${ }^{a}$ Francesco MONTECCHIA ${ }^{a e}$ and Francesco NOZZOLI, ${ }^{a}$}

a Dip. Fisica, Università di Roma "Tor Vergata” and INFN, sez. Roma Tor Vergata, Rome, Italy

${ }^{e}$ Lab. Sperim. Policentrico di Ingegneria Medica, Università di Roma "Tor Vergata”

E-mail: pierluigi.belli@roma2.infn.it

\section{Fabio CAPPELLA, ${ }^{b}$ Annelisa d'ANGELO, ${ }^{b}$ Antonella INCICCHITTI ${ }^{b}$ and Daniele PROSPERI ${ }^{b \dagger}$}

${ }^{b}$ Dip. Fisica, Università di Roma "La Sapienza" and INFN, sez. Roma, Rome, Italy

\section{Riccardo CERULLI, ${ }^{c}$}

${ }^{c}$ Laboratori Nazionali del Gran Sasso, I.N.F.N., Assergi, Italy

\section{C.J. DAI, ${ }^{d}$ H.L. HE, ${ }^{d}$ X.H. MA, ${ }^{d}$ X.D. SHENG, ${ }^{d}$ R.G. WANG ${ }^{d}$ and Z.P. YE ${ }^{d f}$}

${ }^{d}$ IHEP, Chinese Academy, P.O. Box 918/3, Beijing, China

${ }^{f}$ University of Jing Gangshan, Jiangxi, China

The DAMA/LIBRA experiment has a sensitive mass of about $250 \mathrm{~kg}$ highly radiopure $\mathrm{NaI}(\mathrm{Tl})$. It is running at the Gran Sasso National Laboratory of the I.N.F.N. in Italy, and is mainly devoted to the investigation of Dark Matter (DM) particles in the Galactic halo by exploiting the model independent DM annual modulation signature. The present DAMA/LIBRA experiment and the former DAMA/NaI one (the first generation experiment having an exposed mass of about 100 $\mathrm{kg}$ ) have cumulatively released so far the results obtained with the data collected over 13 annual cycles (total exposure: 1.17 ton $\times$ yr). They give a model independent evidence of the presence of DM particles in the galactic halo on the basis of the investigated DM signature at 8.9 $\sigma$ C.L. for the cumulative exposure. Few aspects of the obtained results are summarized and some comments addressed.

\footnotetext{
*Speaker.

$\dagger$ deceased.
} 


\section{Introduction}

DAMA is an observatory for rare processes located deep underground at the Gran Sasso National Laboratory of the I.N.F.N.. It is based on the development and use of low background scintillators. Several set-ups have been realized and are in operation to investigate many rare processes $[1,2,3,4,5,6,7,8,9,10,11,12,13,14,15,16]$.

In particular, DAMA/LIBRA is investigating the presence of Dark Matter (DM) particles in the galactic halo by exploiting the model independent DM annual modulation signature [17]. In fact, as a consequence of its annual revolution around the Sun, which is moving in the Galaxy traveling with respect to the Local Standard of Rest towards the star Vega near the constellation of Hercules, the Earth should be crossed by a larger flux of Dark Matter particles around $\sim 2$ June (when the Earth orbital velocity is summed to the one of the solar system with respect to the Galaxy) and by a smaller one around $\sim 2$ December (when the two velocities are subtracted). This signature offers an efficient model independent signature, able to test a large number of DM candidates, a large interval of cross sections and of halo densities. The DM annual modulation signature is very distinctive since the corresponding signal must simultaneously satisfy all the following requirements: the rate must contain a component modulated according to a cosine function (1) with one year period (2) and a phase that peaks roughly around $\simeq 2^{\text {nd }}$ June (3); this modulation must only be found in a well-defined low energy range, where DM particle induced events can be present (4); it must apply only to those events in which just one detector of many actually "fires" (single-hit events), since the DM particle multi-interaction probability is negligible (5); the modulation amplitude in the region of maximal sensitivity must be $\lesssim 7 \%$ for usually adopted halo distributions (6), but it can be larger in case of some possible scenarios such as e.g. those in Ref. [18, 19, 20]. Only systematic effects or side reactions able to simultaneously fulfil all these requirements and to account for the whole observed modulation amplitude could mimic this signature; thus, no other effect investigated so far in the field of rare processes offers a so stringent and unambiguous signature.

In the following, we will just briefly summarize the results on the DM particle investigation obtained by DAMA/LIBRA ( $\simeq 250 \mathrm{~kg}$ highly radiopure $\mathrm{NaI}(\mathrm{Tl})$ detectors), exploiting over six annual cycles the model independent DM annual modulation signature [15, 21]; the data have also been combined with those previously collected by DAMA/NaI ( $\simeq 100 \mathrm{~kg}$ highly radiopure $\mathrm{NaI}(\mathrm{Tl})$ detectors), over 7 annual cycles.

The DAMA/NaI set up and its performances are reported in Ref. [1, 3, 4, 5], while the DAMA/LIBRA set-up and its performances are described in Ref. [14]. Here we just summarized the main features: i) the sensitive part of the set-up is made of 25 highly radiopure $\mathrm{NaI}(\mathrm{Tl})$ crystal scintillators placed in a 5-rows by 5-columns matrix; ii) the detectors' responses range from 5.5 to 7.5 photoelectrons $/ \mathrm{keV}$; iii) the hardware threshold of each PMT is at single photoelectron (each detector is equipped with two low background photomultipliers working in coincidence); iv) the energy calibration with $\mathrm{X}$-rays $/ \gamma$ sources are regularly carried out down to few $\mathrm{keV}$ in the same conditions as the production runs; v) the software energy threshold of the experiment is $2 \mathrm{keV}$ electron equivalent (hereafter $\mathrm{keV}$ ); vi) both single-hit events (where just one of the detectors fires) and multiple-hit events (where more than one detector fires) are recorded in the production runs; vii) the data are collected up to the $\mathrm{MeV}$ region despite the optimization is performed for the lower one. 


\section{The model independent results}

Details on the collected exposures in each one of the DAMA/LIBRA annual cycles are given in Ref. $[15,21]$. The cumulative DAMA/LIBRA exposure released so far is 0.87 ton $\times \mathrm{yr}(6$ annual cycles), and cumulatively with DAMA/NaI the exposure is 1.17 ton $\times \mathrm{yr}$ (13 annual cycles in total). In particular, in the six DAMA/LIBRA annual cycles about $7.2 \times 10^{7}$ events have been collected for energy calibrations, and about $3 \times 10^{6}$ events $/ \mathrm{keV}$ for the evaluation of the acceptance windows efficiency for noise rejection near energy threshold. In fact, the only data treatment, which is performed on data, is to remove obvious noise pulses (mainly PMT noise, Cherenkov light in the light guides and in the PMT windows, and afterglows) near the energy threshold in the single-hit events; for a description of the used procedure and details see Ref. [14] and Refs. therein. The periodical calibrations and, in particular, those related with the acceptance windows efficiency mainly affect the duty cycle of the experiment.

Several analyses on the model-independent DM annual modulation signature have been performed (see Ref. [15, 21] and references therein); here just few arguments are mentioned. In particular, Fig. 1 shows the time behaviour of the experimental residual rates of the single-hit events collected by DAMA/LIBRA in the (2-4), (2-5) and (2-6) keV energy intervals [15, 21]. The superimposed curves are the cosinusoidal function: $A \cos \omega\left(t-t_{0}\right)$ with a period $T=\frac{2 \pi}{\omega}=1 \mathrm{yr}$, with a phase $t_{0}=152.5$ day (June $2^{\text {nd }}$ ), and modulation amplitude, $A$, obtained by best fit over the 13 cycles of DAMA/NaI [4, 5] and DAMA/LIBRA [15, 21]. The hypothesis of absence of modulation in the data can be discarded [15, 21]. Moreover, when the period and the phase parameters are also released in the fit, values well compatible with those expected for a DM particle induced effect are obtained for the cumulative exposure [21]; for example, the fitting procedure gives in the cumulative (2-6) $\mathrm{keV}$ energy interval: $A=(0.0116 \pm 0.0013) \mathrm{cpd} / \mathrm{kg} / \mathrm{keV}, T=(0.999 \pm 0.002) \mathrm{yr}$ and $t_{0}=(146 \pm 7)$ day. Summarizing, the analysis of the single-hit residual rate favours the presence of a modulated cosine-like behaviour with proper features at $8.9 \sigma$ C.L. [21].

The DAMA/LIBRA single-hit residuals of Fig.1 and those of DAMA/NaI (see e.g. [15]) have also been investigated by a Fourier analysis, obtaining a clear peak corresponding to a period of 1 year (see Fig. 2), as expected for a Dark Matter signal; the same analysis in other energy region shows instead only aliasing peaks. Moreover, while in the (2-6) keV single-hit residuals a clear modulation is present, it is absent at energies just above [21]. The measured energy distribution has been investigated in other energy regions not of interest for Dark Matter, also verifying the absence of any significant background modulation. In fact, the background in the lowest energy region is essentially due to "Compton" electrons, X-rays and/or Auger electrons, muon induced events, etc., which are strictly correlated with the events in the higher energy part of the spectrum; thus, if a modulation detected in the lowest energy region would be due to a modulation of the background (rather than to a signal), an equal or larger modulation in the higher energy regions should be present. The data analyses have allowed one to exclude the presence of a background modulation in the whole energy spectrum at a level much lower than the effect found in the lowest energy region for the single-hit events [21].

A further relevant investigation has been done by applying the same hardware and software procedures, used to acquire and to analyse the single-hit residual rate, to the multiple-hits one. In fact, since the probability that a DM particle interacts in more than one detector is negligible, a DM 
2-4 keV

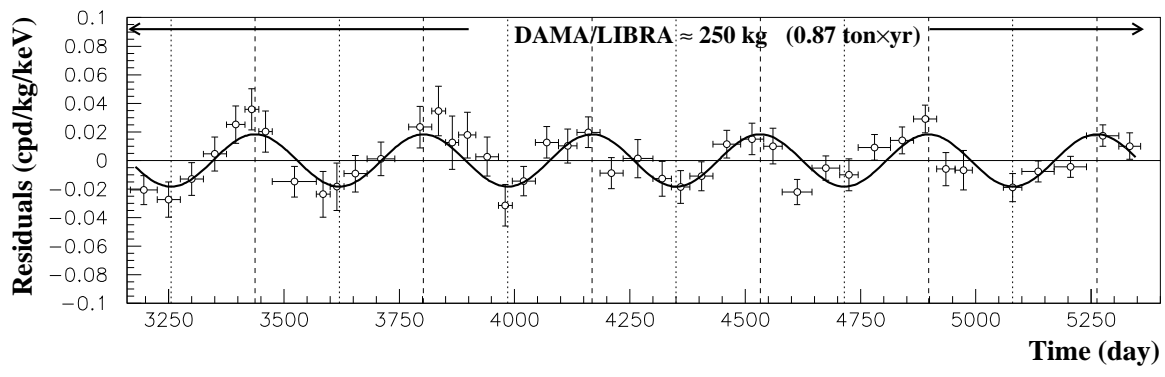

$2-5 \mathrm{keV}$

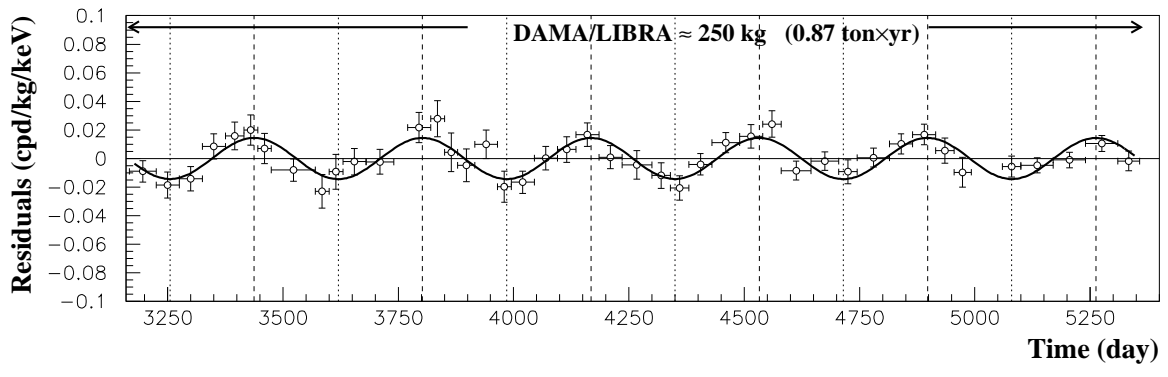

2-6 keV

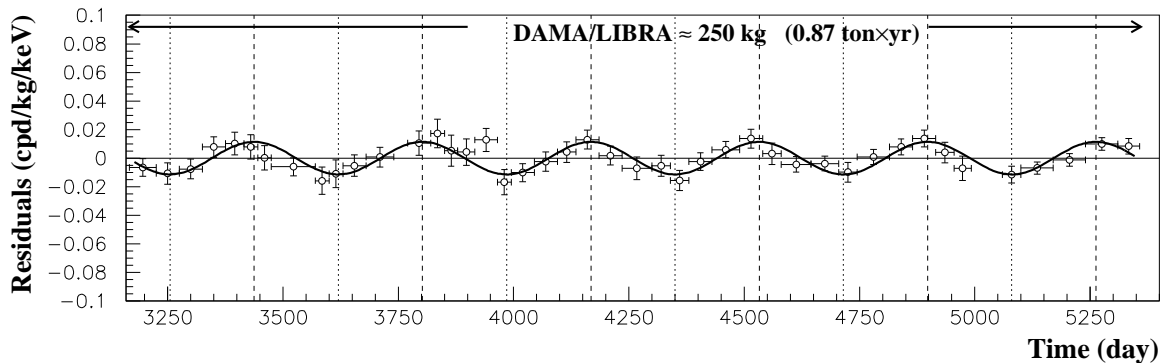

Figure 1: Experimental model-independent residual rate of the single-hit scintillation events, measured by DAMA/LIBRA, 1,2,3,4,5,6 in the $(2-4),(2-5)$ and $(2-6) \mathrm{keV}$ energy intervals as a function of the time $[15,21]$. The zero of the time scale is January $1^{\text {st }}$ of the first year of data taking of the former DAMA/NaI experiment. The experimental points present the errors as vertical bars and the associated time bin width as horizontal bars. The superimposed curves are the cosinusoidal functions behaviors $A \cos \omega\left(t-t_{0}\right)$ with a period $T=\frac{2 \pi}{\omega}=1 \mathrm{yr}$, with a phase $t_{0}=152.5$ day (June $2^{\text {nd }}$ ) and with modulation amplitudes, $A$, equal to the central values obtained by best fit over the whole data including also the exposure previously collected by the former DAMA/NaI experiment: cumulative exposure is 1.17 ton $\times$ yr (see also Ref. [15, 21] and Refs. therein). The dashed vertical lines correspond to the maximum expected for the DM signal (June $2^{\text {nd }}$ ), while the dotted vertical lines correspond to the minimum. See Ref. [15, 21] and text.

signal can be present just in the single-hit residual rate. Thus, this allows the test of the background behaviour in the same energy interval of the observed positive effect. As shown in Fig. 3 a clear modulation is present in the single-hit events, while the fitted modulation amplitude of the multiplehits residual rate is well compatible with zero [21]. Similar results were previously obtained also for the DAMA/NaI case [5]. Thus, again evidence of annual modulation with proper features, as required by the DM annual modulation signature, is present in the single-hit residuals (events class to which the DM particle induced events belong), while it is absent in the multiple-hits residual rate (event class to which only background events belong). Since the same identical hardware and 

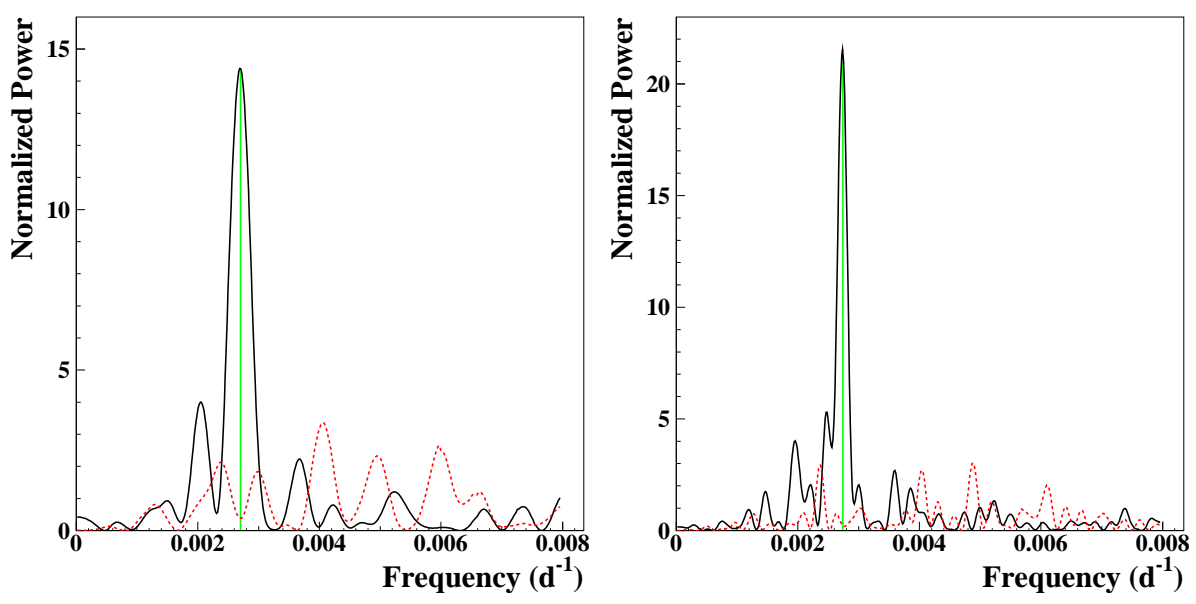

Figure 2: Power spectrum of the measured single-hit residuals in the (2-6) keV (solid lines) and (6-14) $\mathrm{keV}$ (dotted lines) energy intervals calculated according to Ref. [22] including also the treatment of the experimental errors and of the time binning. The data refer to: a) DAMA/LIBRA-1,2,3,4,5,6 (exposure of 0.87 ton $\times \mathrm{yr}$ ); b) the cumulative 1.17 ton $\times$ yr exposure (DAMA/NaI and DAMA/LIBRA-1,2,3,4,5,6). As it can be seen, the principal mode present in the (2-6) keV energy interval corresponds to a frequency of $2.697 \times 10^{-3} \mathrm{~d}^{-1}$ and $2.735 \times 10^{-3} \mathrm{~d}^{-1}$ (vertical lines), respectively in the a) and b) case. They correspond within the uncertainties to a period of 1 year, as expected for a Dark Matter signal. A similar peak is not present in the (6-14) keV energy interval just above. See Ref. [15, 21].

the same identical software procedures have been used to analyse the two classes of events, the obtained result offers an additional strong support for the presence of DM particles in the galactic halo further excluding any side effect either from hardware or from software procedures or from background.

The annual modulation present at low energy has also been analyzed by depicting the differential modulation amplitudes, $S_{m, k}$, as a function of the energy (the $k$ index identifies the energy interval); the $S_{m, k}$ is the modulation amplitude of the modulated part of the signal obtained by maximum likelihood method over the data, considering $T=1 \mathrm{yr}$ and $t_{0}=152.5$ day. The $S_{m, k}$ values are reported as function of the energy in Fig. 4 (for simplicity the $k$ index is omitted in the following). It has been also verified that the measured modulation amplitudes are statistically well distributed in all the crystals, in all the annual cycles and energy bins; these and other discussions can be found in Ref. [21]. It is also interesting the results of the analysis performed by releasing the assumption of a phase $t_{0}=152.5$ day in the procedure of maximum likelihood to evaluate the modulation amplitudes from the data of the seven annual cycles of DAMA/NaI and the six annual cycles of DAMA/LIBRA. In this case alternatively the signal is written as: $S_{0, k}+S_{m, k} \cos \omega\left(t-t_{0}\right)+Z_{m, k} \sin \omega\left(t-t_{0}\right)=S_{0, k}+Y_{m, k} \cos \omega\left(t-t^{*}\right)$, where $S_{0, k}$ is the constant part of the signal in $k$-th energy interval. Obviously, for signals induced by DM particles one would expect: i) $Z_{m, k} \sim 0$ (because of the orthogonality between the cosine and the sine functions); ii) $S_{m, k} \simeq Y_{m, k}$; iii) $t^{*} \simeq t_{0}=152.5$ day. In fact, these conditions hold for most of the dark halo models; however, it is worth noting that slight differences in the phase can be expected in case of possible contributions from non-thermalized DM components, such as e.g. the SagDEG stream [7] and the caustics [23]. The $2 \sigma$ contours in the plane $\left(S_{m}, Z_{m}\right)$ for the (2-6) $\mathrm{keV}$ and 

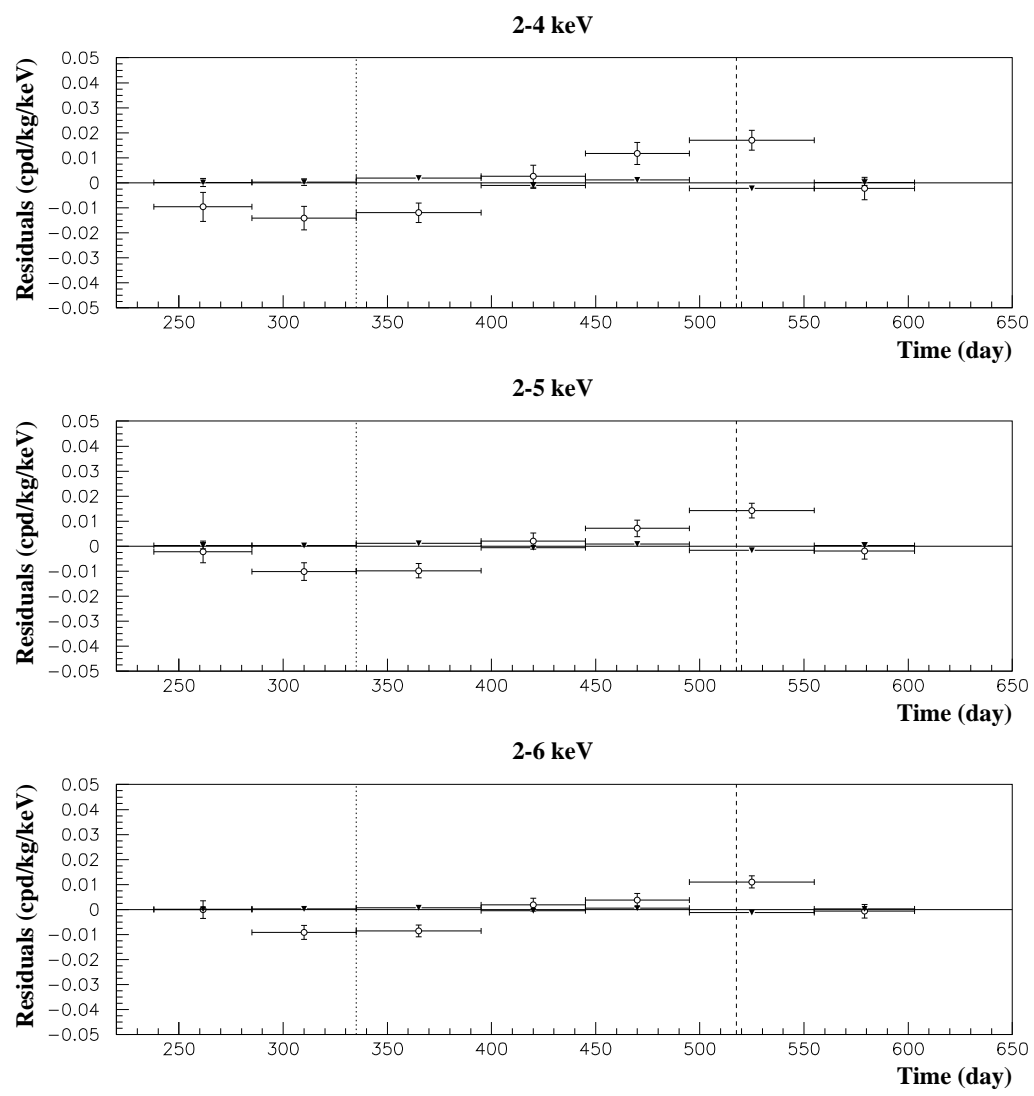

Figure 3: Experimental residual rates over the six DAMA/LIBRA annual cycles for single-hit events (open circles) (class of events to which DM events belong) and for multiple-hit events (filled triangles) (class of events to which DM events do not belong). They have been obtained by considering for each class of events the data as collected in a single annual cycle and by using in both cases the same identical hardware and the same identical software procedures. The initial time of the figure is taken on August $7^{\text {th }}$. The experimental points present the errors as vertical bars and the associated time bin width as horizontal bars. See text and Ref. [15, 21]. Analogous results were obtained for the DAMA/NaI data [5]. See Ref. [15, 21].

(6-14) $\mathrm{keV}$ energy intervals and those in the plane $\left(Y_{m}, t^{*}\right)$ are reported in Fig. 5. The best fit values for the (2-6) keV energy interval are $\left(1 \sigma\right.$ errors): $S_{m}=(0.0111 \pm 0.0013) \mathrm{cpd} / \mathrm{kg} / \mathrm{keV}$; $Z_{m}=-(0.0004 \pm 0.0014) \mathrm{cpd} / \mathrm{kg} / \mathrm{keV} ; Y_{m}=(0.0111 \pm 0.0013) \mathrm{cpd} / \mathrm{kg} / \mathrm{keV} ; t^{*}=(150.5 \pm 7.0)$ day; while for the (6-14) keV energy interval (also shown there) are: $S_{m}=-(0.0001 \pm 0.0008)$ $\mathrm{cpd} / \mathrm{kg} / \mathrm{keV} ; Z_{m}=(0.0002 \pm 0.0005) \mathrm{cpd} / \mathrm{kg} / \mathrm{keV} ; Y_{m}=-(0.0001 \pm 0.0008) \mathrm{cpd} / \mathrm{kg} / \mathrm{keV}$ and $t^{*}$ obviously not determined. These results confirm those achieved by other kinds of analyses. In particular, a modulation amplitude is present in the lower energy intervals for single hit events and the period and the phase agree with those expected for DM induced signals. For more detailed discussions see Ref. [21].

Both the data of DAMA/LIBRA and of DAMA/NaI fulfil all the requirements of the DM annual modulation signature.

As previously done for DAMA/NaI [4, 5], careful investigations on absence of any significant systematics or side reaction effect in DAMA/LIBRA have been quantitatively carried out and re- 


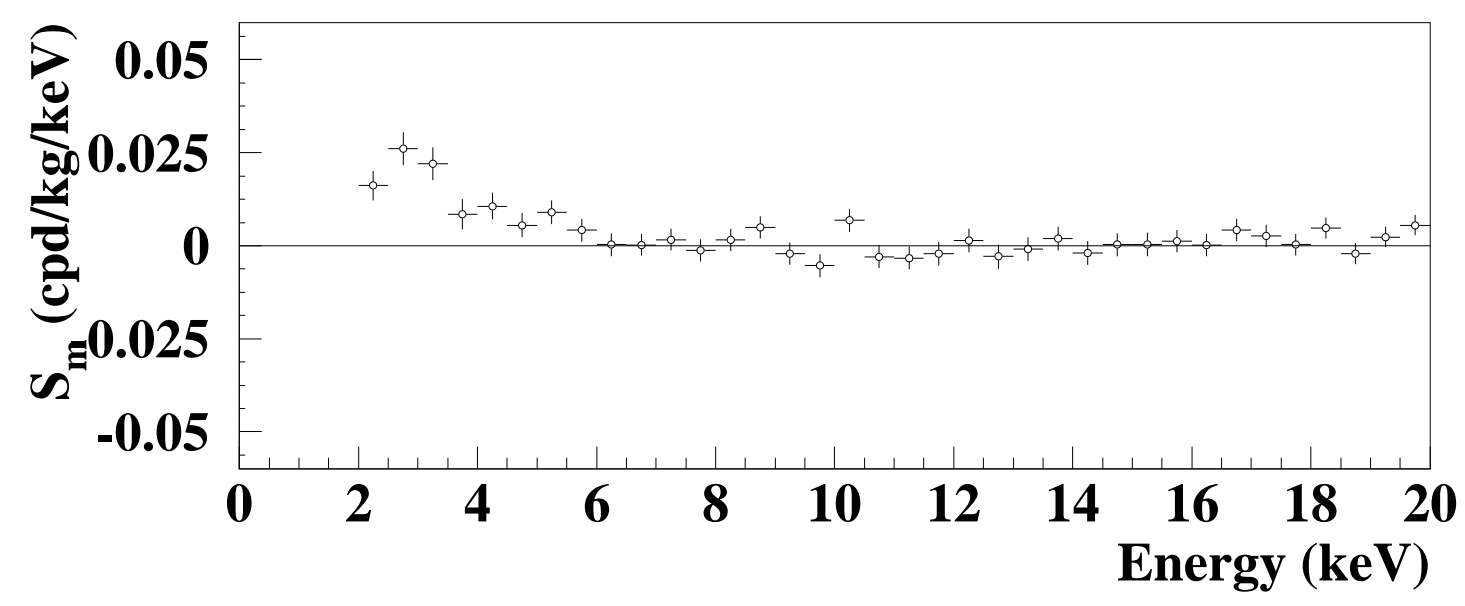

Figure 4: Energy distribution of the $S_{m}$ variable for the total cumulative exposure 1.17 ton $\times$ yr. The energy bin is $0.5 \mathrm{keV}$. A clear modulation is present in the lowest energy region, while $S_{m}$ values compatible with zero are present just above. In fact, the $S_{m}$ values in the (6-20) keV energy interval have random fluctuations around zero with $\chi^{2}$ equal to 27.5 for 28 degrees of freedom. See Ref. [15, 21].
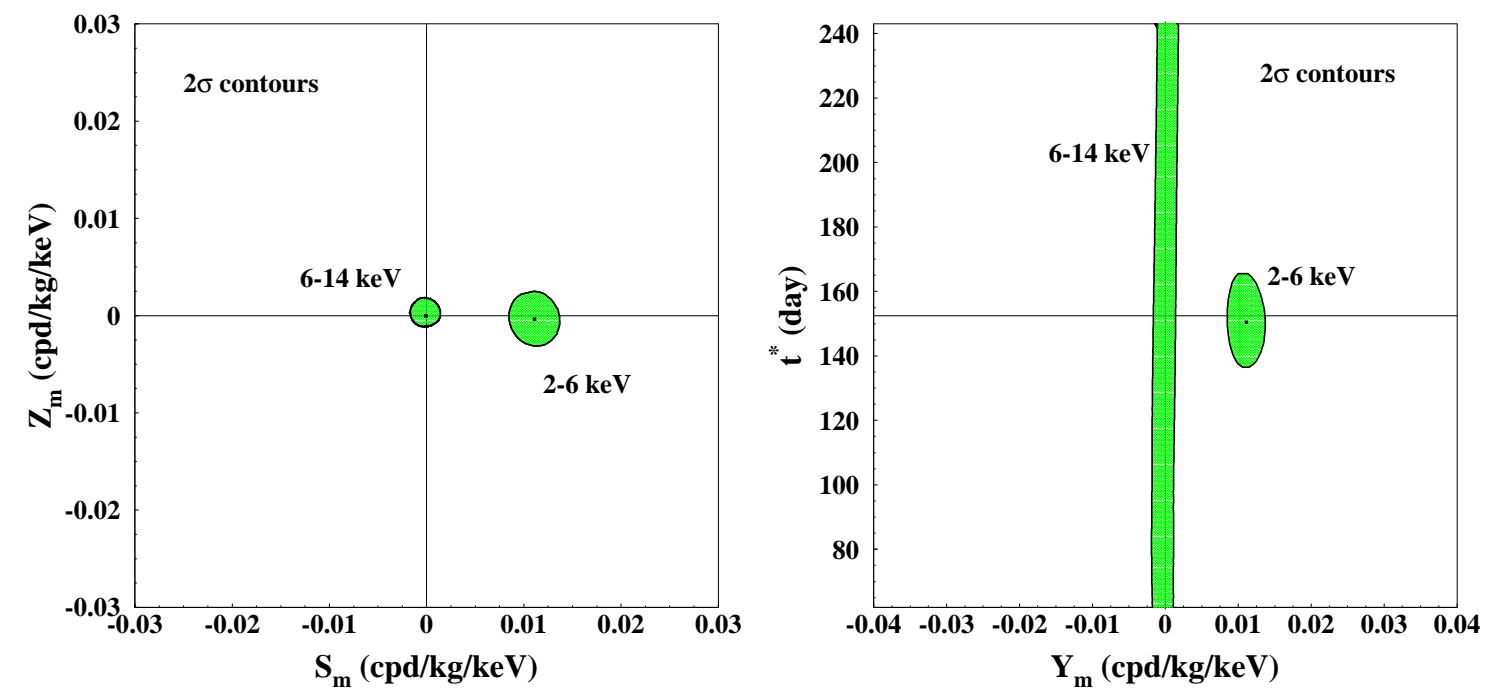

Figure 5: $2 \sigma$ contours in the plane $\left(S_{m}, Z_{m}\right)$ (left) and in the plane $\left(Y_{m}, t^{*}\right)$ (right) for the (2-6) keV and (614) $\mathrm{keV}$ energy intervals. The contours have been obtained by the maximum likelihood method, considering the cumulative exposure of 1.17 ton $\times$ yr. A modulation amplitude is present in the lower energy intervals and the phase agrees with that expected for DM induced signals. See Ref. [15, 21].

ported in details in Ref. [15] and some other arguments have also been addressed in Ref. [24, 25, 26]. No systematics or side reactions able to mimic the signature (that is, able to account for the measured modulation amplitude and simultaneously satisfy all the requirements of the signature) has been found or suggested by anyone over more than a decade. Thus, cautious upper limits $(90 \%$ C.L.) on the possible contributions to the DAMA/LIBRA measured modulation amplitude have been estimated and are summarized in Table 1. For detailed quantitative discussions on all the related topics and for results see Ref. [14, 15, 21, 24, 25, 26] and Refs. therein. 
Table 1: Summary of the results obtained by investigating possible sources of systematics and side processes $[14,15,21,24,25,26]$. None able to give a modulation amplitude different from zero has been found; thus cautious upper limits (90\% C.L.) on the possible contributions to the measured modulation amplitude have been calculated and are shown here for the six annual cycles of DAMA/LIBRA. It is worth noting that none of them is able to mimic the DM annual modulation signature, that is none is able to account for the whole observed modulation amplitude and to contemporaneously satisfy all the requirements of the signature. For details see Ref. [14, 15, 21, 24, 25, 26]. Analogous results were obtained for DAMA/NaI [4, 5].

\begin{tabular}{|c|c|c|}
\hline Source & $\begin{array}{c}\text { Main comment } \\
\text { (also see Ref. [14]) }\end{array}$ & $\begin{array}{l}\text { Cautious upper limit } \\
\text { (90\%C.L.) }\end{array}$ \\
\hline Radon & $\begin{array}{c}\text { Sealed } \mathrm{Cu} \text { Box in } \\
\text { HP Nitrogen atmosphere, } \\
\text { 3-level of sealing }\end{array}$ & $<2.5 \times 10^{-6} \mathrm{cpd} / \mathrm{kg} / \mathrm{keV}$ \\
\hline Temperature & $\begin{aligned} & \text { Air conditioning } \\
+ & \text { huge heat capacity }\end{aligned}$ & $<10^{-4} \mathrm{cpd} / \mathrm{kg} / \mathrm{keV}$ \\
\hline Noise & Efficient rejection & $<10^{-4} \mathrm{cpd} / \mathrm{kg} / \mathrm{keV}$ \\
\hline Energy scale & $\begin{array}{c}\text { Routine } \\
+ \text { intrinsic calibrations }\end{array}$ & $<1-2 \times 10^{-4} \mathrm{cpd} / \mathrm{kg} / \mathrm{keV}$ \\
\hline Efficiencies & Regularly measured & $<10^{-4} \mathrm{cpd} / \mathrm{kg} / \mathrm{keV}$ \\
\hline Background & $\begin{array}{c}\text { No modulation above } 6 \mathrm{keV} \\
\text { no modulation in the }(2-6) \mathrm{keV} \\
\text { multiple-hit events; } \\
\text { this limit includes all possible } \\
\text { sources of background }\end{array}$ & $<10^{-4} \mathrm{cpd} / \mathrm{kg} / \mathrm{keV}$ \\
\hline Side reactions & $\begin{array}{l}\text { From muon flux variation } \\
\text { measured by MACRO }\end{array}$ & $<3 \times 10^{-5} \mathrm{cpd} / \mathrm{kg} / \mathrm{keV}$ \\
\hline \multicolumn{3}{|c|}{ addition: no effect can mimic the signature } \\
\hline
\end{tabular}

In conclusion, the data support over 13 annual cycles the presence of DM particles in the galactic halo on the basis of the DM model independent annual modulation signature at $8.9 \sigma$ C.L. In fact, as required by this DM signature: i) the single-hit events show a clear cosine-like modulation, as expected for the DM signal; ii) the measured phase: (146 \pm 7 ) days, is well compatible with about 152.5 days as expected for the DM signal; iii) the measured period is equal to $(0.999 \pm 0.002)$ $\mathrm{yr}$, well compatible with $1 \mathrm{yr}$ period, as expected for the DM signal; iv) the modulation is present only in the low energy (2-6) keV energy interval and not in other higher energy regions, consistently with the expectation for the DM signal; v) the modulation is present only in the single-hit events, while it is absent in the multiple-hit ones, as expected for the DM signal; vi) the measured modulation amplitude in $\mathrm{NaI}(\mathrm{Tl})$ of the single-hit events in the (2-6) $\mathrm{keV}$ energy interval is: $(0.0116 \pm 0.0013) \mathrm{cpd} / \mathrm{kg} / \mathrm{keV}$ (8.9 $\sigma$ C.L.). No systematics or side process able to simultaneously satisfy all the many peculiarities of the signature and to account for the whole measured modulation amplitude is available nor suggested by anyone over more than a decade. 


\section{Comments}

The obtained model independent evidence - at $8.9 \sigma$ C.L. over 13 annual cycles - is compatible with a wide set of scenarios regarding the nature of the DM candidate and related astrophysical, nuclear and particle Physics. For examples some given scenarios and parameters are discussed in Ref. [2, 4, 5, 6, 7, 8, 9, 10,11] and in Appendix A of Ref. [15]; other arguments can be found in Ref. [27, 28, 18, 29, 30, 20, 31, 32, 33, 34, 35, 36, 37, 38]; others are open.

It is worth recalling that no other experiment exists, whose result can be directly compared in a model-independent way with those by DAMA/NaI and DAMA/LIBRA, and that - more in general - results obtained with different target materials and/or different approaches cannot be directly compared among them in a model-independent way. This is in particular due to the existing experimental and theoretical uncertainties, not last e.g. how many kinds of dark matter particles can exist in the Universe ${ }^{1}$, the nature, the interaction types, the different nuclear and/or atomic correlated aspects, the unknown right halo model, the right DM density, etc. as well as the uncertainties on the values of each one of the many involved experimental and theoretical parameter/assumption/approximation used in the calculations. Moreover, some experimental aspects of some techniques used in the field have also to be addressed [4, 39, 25, 40]. Another relevant argument is the methodological robustness [41]. In particular, the general considerations on comparisons reported in Appendix A of Ref. [15] still hold. Finally, the DAMA regions are generally quoted by others in an incorrect/partial way. Hence, claims for contradiction have no scientific basis.

On the other hand, whatever possible positive hint (as for example those of Ref. [42, 43, 35]) has to be interpreted and a large room of compatibility with DAMA annual modulation evidence is present, and even more when properly considering the existence of large uncertainties in the adopted experimental and theoretical parameters and a right evaluation of the DAMA allowed volumes for each scenario.

Similar considerations can also be done for the indirect detection searches, since it does not exist a biunivocal correspondence between the observables in the direct and indirect experiments. However, if possible excesses in the positron to electron flux ratio and in the $\gamma$ rays flux with respect to some modeling of the background contribution, which is expected from the considered sources, might be interpreted - under some assumptions - in terms of Dark Matter, this would also be not in conflict with the effect observed by DAMA experiments. However, it is worth noting that different possibilities either considering different background modeling or accounting for other kinds of sources can also explain the indirect observations [44].

\section{Conclusion and pespectives}

The data of the former DAMA/NaI and those of the first 6 annual cycles of DAMA/LIBRA (cumulative exposure exposure 1.17 ton $\times \mathrm{yr}$ ) have given positive model independent evidence for

\footnotetext{
${ }^{1}$ In fact, it is worth noting that, considering the richness in particles of the visible matter which is less than $1 \%$ of the Universe density, one could also expect that the particle part of the Dark Matter in the Universe may also be multicomponent.
} 
the presence of DM particles in the galactic halo with high confidence level on the basis of the DM annual modulation signature (see Ref. [15, 21] and references therein). Another year exposure has already been collected. Moreover, the achieved model independent results have pointed out the relevance to lower the energy threshold of the experiment below $2 \mathrm{keV}$. Thus, the replacement of all the PMTs with new ones having higher quantum efficiency is foreseen to be completed at end 2010.

A larger exposure collected by DAMA/LIBRA (or by a possible future DAMA/1ton) and the lowering of the $2 \mathrm{keV}$ energy threshold will further improve the experimental sensitivity and the corollary information on the nature of the DM candidate particle(s) and on the various related astrophysical, nuclear and particle physics scenarios. Moreover, it will also allow the investigation - with high sensitivity - of other DM features, of second order effects and of several rare processes other than DM. In particular, some of the many topics - not yet well known at present and which can affect whatever model dependent result and comparison - are the studies about: i) the velocity and spatial distribution of the Dark Matter particles in the galactic halo, ii) the effects induced on the Dark Matter particles distribution in the galactic halo by contributions from satellite galaxies tidal streams; iii) the effects induced on the Dark Matter particles distribution in the galactic halo by the possible existence of caustics; iv) the detection of possible "solar wakes" (the gravitational focusing effect of the Sun on the Dark Matter particle of a stream). Finally, other arguments are the investigation of possible diurnal effects, the study of possible structures as clumpiness with small scale size, the coupling(s) of the Dark Matter particle with the ${ }^{23} \mathrm{Na}$ and ${ }^{127} \mathrm{I}$ and its nature, the scaling laws and cross sections, etc.

In addition, it is worth noting that ultra low background $\mathrm{NaI}(\mathrm{Tl})$ scintillators can also offer the possibility to achieve significant results on several other rare processes as already done e.g. by the former DAMA/NaI apparatus [12] and just started with DAMA/LIBRA [16].

Finally, we mention that a third generation R\&D effort towards a possible NaI(Tl) ton set-up, DAMA proposed in 1996, is in progress.

\section{References}

[1] R. Bernabei et al., Il Nuovo Cim. A 112 (1999) 545-576.

[2] R. Bernabei et al., Phys. Lett. B 389 (1996) 757-766; R. Bernabei et al., Phys. Lett. B 424 (1998) 195-201; R. Bernabei et al., Phys. Lett. B 450 (1999) 448-455; P. Belli et al., Phys. Rev. D 61 (2000) 023512-9; R. Bernabei et al., Phys. Lett. B 480 (2000) 23-31; ; R. Bernabei et al., Phys. Lett. B 509 (2001) 197-203; R. Bernabei et al., Eur. Phys. J. C 23 (2002) 61-64; P. Belli et al., Phys. Rev. D 66 (2002) 043503-17.

[3] R. Bernabei et al., Eur. Phys. J. C 18 (2000) 283-292.

[4] R. Bernabei el al., La Rivista del Nuovo Cimento 26 n.1 (2003) 1-73.

[5] R. Bernabei et al., Int. J. Mod. Phys. D 13 (2004) 2127-2159.

[6] R. Bernabei et al., Int. J. Mod. Phys. A 21 (2006) 1445-1469.

[7] R. Bernabei et al., Eur. Phys. J. C 47 (2006) 263-271.

[8] R. Bernabei et al., Int. J. Mod. Phys. A 22 (2007) 3155-3168.

[9] R. Bernabei et al., Eur. Phys. J. C 53 (2008) 205-213. 
[10] R. Bernabei et al., Phys. Rev. D 77 (2008) 023506-9.

[11] R. Bernabei et al., Mod. Phys. Lett. A 23 (2008) 2125-2140.

[12] R. Bernabei et al., Phys. Lett. B 408 (1997) 439-444; P. Belli et al., Phys. Lett. B 460 (1999) 236-241; R. Bernabei et al., Phys. Rev. Lett. 83 (1999) 4918-4921; P. Belli et al., Phys. Rev. C 60 (1999) 065501-7; R. Bernabei et al., Il Nuovo Cimento A 112 (1999) 1541-1552; R. Bernabei et al., Phys. Lett. B 515 (2001) 6-12; F. Cappella et al., Eur. Phys. J.-direct C 14 (2002) 1-6; R. Bernabei et al., Eur. Phys. J. A 23 (2005) 7-10; R. Bernabei et al., Eur. Phys. J. A 24 (2005) 51-56; R. Bernabei et al., Astrop. Phys. 4 (1995) 45-54; R. Bernabei, in The identification of Dark Matter, World Sc. Pub., Singapore, 1997, pp. 574.

[13] DAMA web page: http://people.roma2.infn.it/dama

[14] R. Bernabei et al., Nucl. Instr. \& Meth. A 592 (2008) 297-315.

[15] R. Bernabei et al., Eur. Phys. J. C 56 (2008) 333-355 [arXiv: 0804 . 2741].

[16] R. Bernabei et al., Eur. Phys. J. C 62 (2009) 327-332.

[17] K. A. Drukier et al., Phys. Rev. D 33 (1986) 3495-3508; K. Freese et al., Phys. Rev. D 37 (1988) 3388-3405.

[18] D. Smith and N. Weiner, Phys. Rev. D 64 (2001) 043502; D. Tucker-Smith and N. Weiner, Phys. Rev. D 72 (2005) 063509; D. P. Finkbeiner et al, Phys. Rev. D 80 (2009) 115008.

[19] K. Freese et al., Phys. Rev. D 71 (2005) 043516-15; K. Freese et al., Phys. Rev. Lett. 92 (2004) $111301-4$.

[20] Y. Bai and P.J. Fox, arXiv:0909.2900.

[21] R. Bernabei et al., Eur. Phys. J. C 67 (2010) 39.

[22] W.H. Press and G. B. Rybicki, Astrophys. J. 338 (1989) 277; J.D. Scargle, Astrophys. J. 263 (1982) 835.

[23] F. S. Ling, P. Sikivie and S. Wick, Phys. Rev. D 70 (2004) 123503-19.

[24] R. Bernabei et al., AIP Conf. Proceed. 1223 (2010) 50 [arXiv: 0912 . 0660].

[25] R. Bernabei et. al, J. Phys.: Conf. Ser. 203 (2010) 012040 [arXiv : 0912 . 42 00]; http://taup2009.lngs.infn.it/slides/jul3/nozzoli.pdf, talk given by F. Nozzoli.

[26] R. Bernabei et. al, arXiv:1007.0595.

[27] M. W. Goodman and E. Witten, Phys. Rev. D 31 (1985) 3059; W. H. Press and D. N. Spergel, Astrophys. J. 296 (1985) 679; R. L. Gilliland et al., Astrophys. J. 306 (1986) 703; K. Griest, Phys. Rev. D 38 (1988) 2357; K. Griest, Phys. Rev. Lett. 61 (1988) 666; R. Barbieri, M. Frigeni and G.F. Giudice, Nucl. Phys. B 313 (1989) 725; G. Gelmini, P. Gondolo and E. Roulet, Nucl. Phys. B 351 (1991) 623; A. Bottino, V. de Alfaro, N. Fornengo, G. Mignola and S. Scopel, Astrop. Phys. 2 (1994) 77.

[28] A. Bottino et al., Phys. Lett. B 402 (1997) 113; A. Bottino et al., Phys. Lett. B 423 (1998) 109; A. Bottino et al., Phys. Rev. D 59 (1999) 095004; A. Bottino et al., Phys. Rev. D 59 (1999) 095003; A. Bottino et al., Astrop. Phys. 10 (1999) 203; A. Bottino et al., Astrop. Phys. 13 (2000) 215; A. Bottino et al., Phys. Rev. D 62 (2000) 056006; A. Bottino et al., Nucl. Phys. B 608 (2001) 461; A. Bottino, F. Donato, N. Fornengo and S. Scopel, Phys. Rev. D 63 (2001) 125003; A. Bottino et al., Phys. Rev. D 67 (2003) 063519; A. Bottino et al., Phys. Rev. D 68 (2003) 043506; A. Bottino et al., Phys. Rev. D 69 (2004) 037302; T. Asaka et al., Phys. Rev. D 58 (1998) 023507; T. Asaka et al., Phys. Rev. D 58 (1998) 
083509; R. Volkas, Prog. Part. Nucl. Phys. 48 (2002) 161; R. Foot, hep-ph/ 0308254 ; K. Belotsky, D. Fargion, M. Khlopov, R.V. Konoplich, hep-ph/ 0411093 ; D. Hooper and L. T. Wang, Phys. Rev. D 70 (2004) 063506; S. Mitra, Phys. Rev. D 71 (2005) 121302(R); C. Picciotto and M. Pospelov, Phys. Lett. B 605 (2005) 15; M. Kawasaki and T. Yanagida, Phys. Lett. B 624 (2005) 162; D. V. Ahluwalia-Khalilova and D. Grumiller, Phys. Rev. D 72 (2005) 067701; D. V. Ahluwalia-Khalilova and D. Grumiller, JCAP 07 (2005) 012; J. Knodlseder et al., Astron. Astrophys. 441 (2005) 513; J. M. Frére et al., arXiv : hep-ph / 610240 ; Y. Ascasibar, P. Jean, C. Boehm, and J. Knoedlseder, Mon. Not. R. Astron. Soc. 368 (2006) 1695; E.M. Drobyshevski et al., Astron. \& Astroph. Trans. 26:4 (2007) 289; E.M. Drobyshevski et al., arXiv:0704.0982 and arXiv:0706.3095; D. Hooper et al., arXiv: 0704.2558 [astro-ph] ; C. Jacoby and S. Nussinov, J. High Energy Phys. 05 (2007) 017; D. P. Finkbeiner and N. Weiner, Phys. Rev. D 76 (2007) 083519; M. Pospelov and A. Ritz, Phys. Lett. B 651 (2007) 208; P. Fayet, Phys. Rev. D 75 (2007) 115017; A. Kusenko, AIP Conf. Proc. 917 (2007) 58; A. Palazzo et al., Phys. Rev. D 76 (2007) 103511; M. Shaposhnikov, Nucl. Phys. B 763 (2007) 49; M. Lemoine et al., Phys. Lett. B 645 (2007) 222; C. Arina and N. Fornengo, arXiv:0709.4477; A. Bottino et al., Phys. Rev. D 77 (2008) 015002.

[29] A. Bottino, N. Fornengo, and S. Scopel, Phys. Rev. D 67 (2003) 063519; A. Bottino, F. Donato, N. Fornengo, and S. Scopel, Phys. Rev. D 69 (2003) 037302; A. Bottino et al., Phys. Rev. D 78 (2008) 083520; A. Bottino, F. Donato, N. Fornengo, S. Scopel, Phys. Rev. D 81 (2010) 107302 [arXiv:0912.4025]; N. Fornengo, S. Scopel, A. Bottino, arXiv:1011.4743.

[30] R. Foot, Phys. Rev. D 78 (2008) 043529.

[31] K. Belotsky, D. Fargion, M. Khlopov and R.V. Konoplich, Phys. Atom. Nucl. 71 (2008) 147.

[32] E.M. Drobyshevski et al., Astrohys. \& Astronom. Trans. 26:4 (2007) 289; Mod. Phys. Lett. A 23 (2008) 3077.

[33] Nima Arkani-Hamed et al., Phys. Rev. D 79 (2009) 015014.

[34] Daniele S.M. Alves et al., arXiv:0903.3945.

[35] D. Hooper, L. Goodenough, arXiv:1010.2752; D. Hooper et al., arXiv:1007.1005.

[36] S. Chang, R. F. Lang, N. Weiner, arXiv:1007.2688.

[37] S. Chang, N. Weiner, I. Yavin, arXiv: 1007.4200.

[38] S. Andreas, A. Ringwald, arXiv: 1008.4519.

[39] R. Bernabei et al., Liquid Noble gases for Dark Matter searches: a synoptic survey, Exorma Ed., Roma, ISBN 978-88-95688-12-1, 2009, pp. 1-53 [arXiv: $0806.0011 v 2]$.

[40] J.I. Collar and D.N. McKinsey, arXiv: 1005 . 0838 ; J.I. Collar and D.N. McKinsey, arXiv:1005.3723; J.I. Collar, arXiv:1006.2031; J.I. Collar, arXiv:1010.5187.

[41] R. Hudson, Found. Phys. 39, 174-193 (2009).

[42] C.E. Aalseth et al., arXiv:1002.4703.

[43] W. Seidel (CRESST collaboration), presented at the 8th Intl. Workshop on Identification of Dark Matter, Montpellier, July 2010.

[44] F. Donato et al., Phys. Rev. Lett. 102 (2009) 071301; T. Delahaye et al., Astron. Astrophys. 501 (2009) 821; S. Profumo, arXiv: 0812 . 44 57; P. Blasi, Phys. Rev. Lett. 103 (2009) 051104; M. Ahlers et al., arXiv:0909.4060. 